\title{
Influência da composição granulométrica das partículas de resíduos de madeira nas propriedades de compósitos minerais: parte 2: gesso-madeira
}

\author{
Influence of particle size of wood residues on the \\ properties of mineral composites: part 2: gypsum-wood
}

\section{Thaísa Mariana Santiago Rocha \\ Leonardo Fagundes Rosemback Miranda Carlos Frederico Alice Parchen}

\section{Resumo}

esíduos de construção e demolição (RCD) de madeira podem ser incorporados em compósitos de fibras naturais, reduzindo os impactos ambientais, o que contribui para o desenvolvimento de materiais, produtos e processos mais sustentáveis. Nesse sentido, o objetivo deste estudo foi avaliar a influência da composição granulométrica das partículas de madeira, provenientes de RCD, nas propriedades físicas e mecânicas de compósitos de gesso-madeira. As partículas de madeira (compensado, eucalipto e pínus) foram moídas em moinho de martelos em diferentes tempos e caracterizadas. Foi utilizado, também, o gesso de pega lenta e a água. Os compósitos de gesso-madeira foram produzidos com diferentes relações aglomerante/madeira com relação água/gesso fixada em 0,70 em massa. Como conclusão, tem-se que os compósitos produzidos com a composição P1,2, ou com compensado ou pínus, ou com as proporções $11 \%$ ou $15 \%$ apresentam os menores valores de densidade de massa endurecida. Enquanto os compósitos produzidos com a composição P1, ou com eucalipto, ou com a proporção de 7,5\% apresentam maior resistência à tração na flexão e à compressão.

Palavras-chave: Resíduos de Construção e Demolição (RCD). Compósitos. Partículas de madeira. Composição granulométrica. Gesso.

${ }^{1}$ Thaísa Mariana Santiago Rocha ${ }^{1}$ Universidade Federal do Paraná Curitiba - PR - Brasil https:// orcid. org/ 0000-0002-8586-6449

${ }^{2}$ Leonardo Fagundes Rosemback Miranda

2Universidade Federal do Paraná Curitiba - PR - Brasil

${ }^{3}$ Carlos Frederico Alice Parchen ${ }^{3}$ Universidade Federal do Paraná Curitiba - PR - Brasil

Recebido em 27/08/18 Aceito em 14/12/18

\begin{abstract}
Construction and demolition waste (CDW) wood can be incorporated into natural fibre composites, reducing environmental impacts, contributing to the development of more sustainable materials, products, and processes. The objective of this study was to evaluate the influence of particle size distribution of wood particles from construction and demolition residues (RCD) on the physical and mechanical properties of gypsum-wood composites. The wood particles (plywood, eucalyptus spp. and pinus spp.) were milled with hammers at different times and then characterised. Slow paste gypsum and water were also used. The gypsum-wood composites were produced with different binder/wood ratios in relation to water/gypsum set at 0.70 by mass. The study concludes that composites produced with the composition $P 1,2$, either with plywood or pinus, or with the proportions $11 \%$ or $15 \%$ present the lowest hardened bulk density values, while the composites produced with the composition $\mathrm{P} 1$, or with eucalyptus, or with the proportion of $7.5 \%$ present greater flexural and compression strength.
\end{abstract}

Keywords: Construction and Demolition Waste (CDW). Composites. Wood particles. Particle size. Gypsum. 


\section{Introdução}

O setor da construção civil consome grande quantidade de energia, além de provocar diversos impactos ao ambiente, como a emissão de gases do efeito estufa decorrentes da produção do cimento, o consumo de água e a geração de resíduos sólidos (ASHOUR et al., 2015). Além disso, o elevado consumo dos recursos naturais e, consequentemente, o esgotamento destes têm contribuído para a utilização de matérias-primas renováveis para a produção de materiais de construção (AGOUA et al., 2013; AKINYEMI; AFOLAYAN; OLUWATOBI, 2016; FARAHANI et al., 2017), e, por isso, agregados minerais têm sido substituídos por materiais alternativos como, por exemplo, resíduos de madeira (CORINALDESI; MAZZOLI; SIDDIQUE, 2016; OSSA; GARCÍA; BOTERO, 2016; RUMSYS et al., 2017).

A geração de RCD de madeira está diretamente relacionada ao processo construtivo realizado em determinada região (PEDREÑO-ROJAS et al., 2017), podendo atingir uma média de 2,6\% na União Europeia e 10,12\% na Noruega do total de resíduo produzido (EUROSTAT, 2017) e, por isso, torna-se imprescindível seu reaproveitamento.

Os compósitos de fibras naturais podem ser uma alternativa, principalmente em países em desenvolvimento, sendo capazes de absorver os resíduos, reduzindo os impactos ambientais (KHORAMI; GANJIAN, 2013), além de contribuir para o desenvolvimento de materiais, produtos e processos mais sustentáveis (AKIL et al., 2011; VÄNTSI; KÄRKI, 2015). Ademais, as fibras naturais têm um excelente potencial para reduzir as emissões de $\mathrm{CO}_{2}$ e diminuir o consumo de recursos não renováveis (PERVAIZ; SAIN, 2003), podendo proporcionar benefícios ambientais, quando comparadas à utilização de fibras poliméricas, confirmados através de análises de ciclo de vida (PIETRINI et al., 2007; XU et al., 2008; MILLER; LEPECH; BILLINGTON, 2013), além de apresentar viabilidade técnica e econômica (SAVASTANO JUNIOR et al., 1999).

Os compósitos minerais associados às fibras lignocelulósicas geralmente são produzidos a partir da mistura de um aglomerante mineral com as partículas de madeira. Dentre os aglomerantes utilizados, os principais são o gesso, o cimento de magnésio e o cimento Portland (FOREST..., 2010). Entretanto, na presença de cimento, as fibras lignocelulósicas apresentam efeitos deletérios sobre o aglomerante, como, por exemplo, forte efeito de retardamento de pega (MEHTA; MONTEIRO,
2014). Diante disso, o gesso surge como alternativa, contribuindo para o meio ambiente devido à redução das emissões de $\mathrm{CO}_{2}$ quando comparado à produção do cimento Portland (MAGALLANESRIVERA et al., 2012; TAN et al., 2018), e com o desenvolvimento de compostos ecoeficientes quando realizada a adição de resíduos de madeira numa matriz de gesso (PEDREÑO-ROJAS et al., 2017).

Nesse sentido, o objetivo deste estudo (Parte 2) é avaliar a influência da composição granulométrica das partículas de madeira, provenientes de RCD, nas propriedades físicas e mecânicas de compósitos de gesso-madeira.

\section{Programa experimental}

Foram produzidos compósitos de gesso-madeira em que as partículas de madeira são provenientes de RCD. Os resíduos de compensado, eucalipto e pínus foram moídos e classificados quanto à granulometria. Os compósitos produzidos foram avaliados com relação às propriedades no estado fresco e no estado endurecido aos 28 dias.

\section{Materiais}

Foi utilizado como aglomerante o gesso de pega lenta caracterizado quanto à composição química por meio do ensaio de fluorescência de raios-X (FRX) (Tabela 1) e com massa unitária de 0,84 $\mathrm{g} / \mathrm{cm}^{3}$.

Foram obtidos três tipos diferentes de madeira provenientes de RCD, sendo: compensado, eucalipto e pínus (Figura 1). Os resíduos de madeira foram coletados em canteiros de obras situados nos estados do Paraná e de Santa Catarina. Estes foram moídos em um moinho de martelos portátil com potência de 5,0 cv e 1.160 RPM.

O procedimento de moagem realizado foi o mesmo para todas as composições granulométricas apresentadas e classificadas neste estudo (Tabela 2). O tempo que o resíduo levou para percorrer o moinho de martelos e originar as partículas foi de 3 segundos. Entretanto, as composições granulométricas identificadas como P1 foram submetidas a apenas uma repetição do procedimento de moagem. As composições P3 foram moídas por três vezes consecutivas. Por fim, as composições granulométricas identificadas como $\mathrm{P} 1,2$ referem-se às partículas moídas e obtidas a partir do passante na peneira 1,2 $\mathrm{mm}$. 


\begin{tabular}{c|c|c|c|c|c|c|c|c}
\hline \multicolumn{10}{c}{ Composição química } \\
\hline $\mathrm{SO}_{3}(\%)$ & $\mathrm{CaO}(\%)$ & $\mathrm{SiO}_{2}(\%)$ & $\mathrm{MgO}(\%)$ & $\mathrm{Al}_{2} \mathrm{O}_{3}(\%)$ & $\mathrm{SrO}(\%)$ & $\mathrm{Fe}_{2} \mathrm{O}_{3}(\%)$ & $\mathrm{K}_{2} \mathrm{O}(\%)$ & P.F. (\%) \\
51 & 42,7 & 0,3 & 0,1 & 0,1 & 0,1 & $<0,1$ & $<0,1$ & 5,63 \\
\hline
\end{tabular}

\section{Figura 1 - Preparo, coleta e moagem de RCD de madeira}

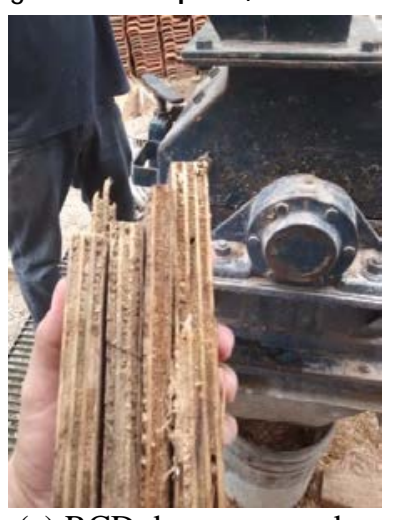

(a) RCD de compensado

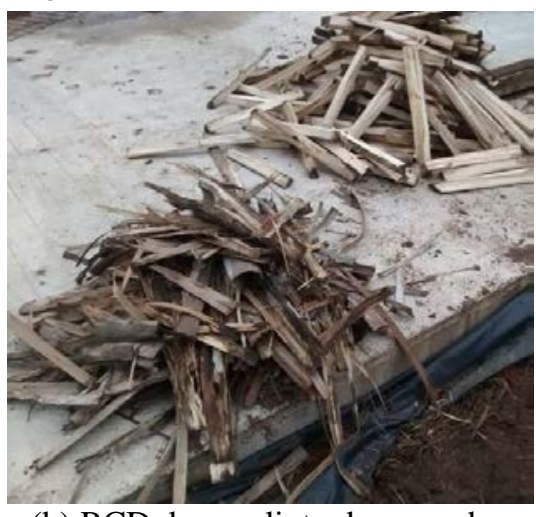

(b) RCD de eucalipto descascado

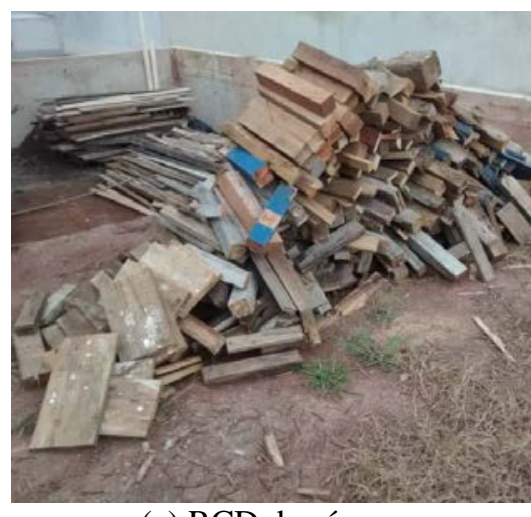

(c) RCD de pínus

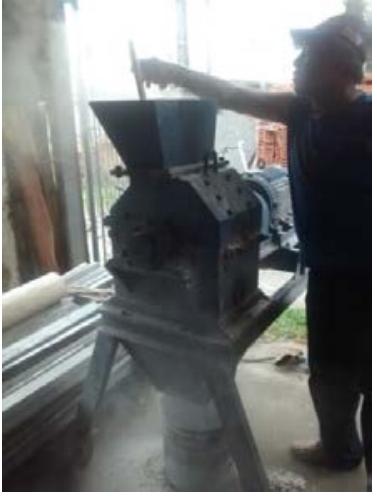

(d) Moinho de martelos utilizado para a moagem do RCD

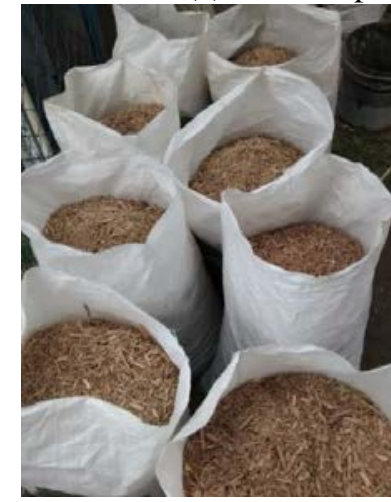

(e) Partículas de madeira provenientes de RCD

Fonte: Rocha (2017).

Tabela 2 - Identificação das partículas de madeira

\begin{tabular}{c|l}
\hline Identificação & \multicolumn{1}{c}{ Características } \\
\hline P1 & Partículas geradas após a moagem realizada uma vez (1x) no moinho \\
P3 & Partículas geradas após a moagem realizada três vezes (3x) no moinho \\
P1,2 & Partículas geradas a partir do passante na peneira 1,2 mm \\
\hline
\end{tabular}

A identificação e a classificação das diferentes composições granulométricas das partículas de madeira foram realizadas no intuito de viabilizar a verificação da influência destas nos compósitos cimentícios. A repetição da moagem foi realizada para que fosse possível obter o volume necessário de cada granulometria das partículas de madeira para a realização dos ensaios.

\section{Método}

O pré-tratamento das partículas de madeira foi realizado em água fria por 48 h numa proporção de 1:10 (madeira:água), em seguida, as partículas foram secas ao ar por 72 h, para que então fosse realizada a secagem em estufa a $80^{\circ} \mathrm{C}$ por $24 \mathrm{~h}$. Para a produção dos compósitos, a relação água/gesso foi fixada em 0,70 (em massa). Como as partículas encontravam-se secas, foi aplicada a compensação de água proposta por Simatupang (1979) (Equação 1).

$C a=R_{a / a} \times C+(P S F-U) \times M \quad$ Eq. 1

Onde:

Ca = Consumo de água (g);

$R_{a / a}=$ Relação água/aglomerante; 
$C=$ Consumo de aglomerante (g);

$P S F=$ Ponto de saturação das fibras (adotado como 30\%);

$U=$ Teor de umidade da madeira (\%); e

$M=$ Massa de madeira (g).

\section{Análise comparativa das composições granulométricas das partículas de madeira}

Para comparar a composição granulométrica das partículas obtidas após a moagem, foram realizados os ensaios de caracterização para a determinação da massa unitária C29 (AMERICAN..., 2017), da composição granulométrica C136 (AMERICAN..., 2014a) e do teor de umidade D4442 (AMERICAN..., 2016). O teor de umidade das partículas de madeira foi utilizado para determinar a quantidade de água adicionada na mistura, conforme descrito na Equação 1.

\section{Análise da influência das composições granulométricas das partículas no estado fresco}

Para avaliar a influência das composições granulométricas das partículas no estado fresco foram produzidos compósitos (Tabela 3) e realizados os ensaios de determinação do tempo de pega C472 (AMERICAN..., 2014b) e de densidade de massa fresca C270 (AMERICAN..., 2014c). A proporção aglomerante/madeira foi de 1:0,075 (em massa), conforme proposto por Moslemi, Garcia e Hofstrand (1983), Latorraca, Iwakiri e Lelis (1999), Latorraca (2000), Lima (2009) e Lima e Iwakiri (2011). Essa proporção será considerada de referência, sendo identificada por 7,5\%.

\section{Análise da influência das composições granulométricas das partículas no estado fresco}

Para avaliar a influência das composições granulométricas das partículas no estado endurecido foram produzidos corpos de provas prismáticos $\left(4 \mathrm{~cm}^{3} \times 4 \mathrm{~cm}^{3} \times 16 \mathrm{~cm}^{3}\right)$ (Tabela 4), curados em câmara seca (a $21{ }^{\circ} \mathrm{C} \pm 2{ }^{\circ} \mathrm{C}$ e com umidade inferior a 50\%), para que, aos 28 dias, fossem realizados os ensaios de densidade de massa endurecida, de resistência à tração na flexão C348 (AMERICAN..., 2014d) e à compressão C349 (AMERICAN..., 2014e).

Nessa etapa, além da proporção aglomerante/madeira de $7,5 \%$ e a amostra de referência ( $0 \%$ de partículas de madeira), foram produzidos também compósitos nas proporções $11 \%(1: 0,11)$ e $15 \%(1: 0,15)$, no intuito de avaliar também a influência dos maiores teores de partículas de madeira nas propriedades mecânicas dos compósitos. Não foram realizados os ensaios no estado fresco para as proporções aglomerante/madeira de $11 \%$ e $15 \%$. Isso porque a quantidade de partículas na mistura impediu a execução adequada do ensaio de tempo de início e de fim de pega, mascarando os resultados. Além disso, é esperado que uma maior quantidade de mistura na composição contribua para a diminuição do índice de consistência e da densidade de massa endurecida.

O delineamento estatístico utilizado foi análise fatorial com ênfase na natureza dos tratamentos, sendo estes: a composição granulométrica das partículas (P1, P3 e P1,2), o tipo de partícula (compensado, eucalipto e pínus) e a proporção de madeira na composição (7,5\% - 11\% - 15\%). Para a comparação entre as médias, utilizou-se ANOVA em conjunto com o teste de Tukey, no nível de probabilidade de $95 \%$.

Tabela 3 - Composição dos compósitos de madeira produzidos para avaliação no estado fresco

\begin{tabular}{|c|c|c|c|c|}
\hline \multicolumn{3}{|c|}{ Identificação } & $\begin{array}{c}\text { Relação água/aglomerante } \\
\text { (em massa) }\end{array}$ & $\begin{array}{c}\text { Proporção aglomerante/ } \\
\text { madeira (em massa) }\end{array}$ \\
\hline \multirow{10}{*}{ 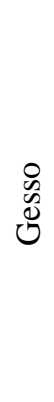 } & & Compensado & \multirow{9}{*}{0,70} & \multirow{9}{*}{$7,5 \%$} \\
\hline & $\mathrm{P} 1$ & Eucalipto & & \\
\hline & & Pínus & & \\
\hline & \multirow{3}{*}{ P3 } & Compensado & & \\
\hline & & Eucalipto & & \\
\hline & & Pínus & & \\
\hline & \multirow{3}{*}{$\begin{array}{c}\mathrm{P} 1, \\
2\end{array}$} & Compensado & & \\
\hline & & Eucalipto & & \\
\hline & & Pínus & & \\
\hline & & Ref & 0,70 & $0 \%$ \\
\hline
\end{tabular}


Tabela 4 - Composição dos compósitos de madeira produzidos para avaliação no estado endurecido

\begin{tabular}{|c|c|c|c|c|}
\hline \multicolumn{3}{|c|}{ Identificação } & $\begin{array}{c}\text { Relação água/ } \\
\text { aglomerante (em massa) }\end{array}$ & $\begin{array}{c}\text { Proporção aglomerante/ } \\
\text { madeira (em massa) }\end{array}$ \\
\hline \multirow{28}{*}{ 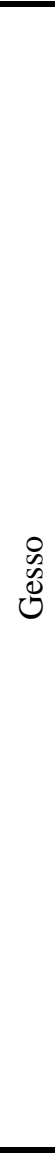 } & \multirow{3}{*}{$\mathrm{P} 1$} & Compensado & \multirow{9}{*}{0,70} & \multirow{9}{*}{$7,5 \%$} \\
\hline & & Eucalipto & & \\
\hline & & Pínus & & \\
\hline & \multirow{3}{*}{ P3 } & Compensado & & \\
\hline & & Eucalipto & & \\
\hline & & Pínus & & \\
\hline & \multirow{3}{*}{$\mathrm{P} 1,2$} & Compensado & & \\
\hline & & Eucalipto & & \\
\hline & & Pínus & & \\
\hline & \multirow{3}{*}{ P1 } & Compensado & \multirow{9}{*}{0,70} & \multirow{9}{*}{$11 \%$} \\
\hline & & Eucalipto & & \\
\hline & & Pínus & & \\
\hline & \multirow{3}{*}{ P3 } & Compensado & & \\
\hline & & Eucalipto & & \\
\hline & & Pínus & & \\
\hline & \multirow{3}{*}{$\mathrm{P} 1,2$} & Compensado & & \\
\hline & & Eucalipto & & \\
\hline & & Pínus & & \\
\hline & \multirow{3}{*}{ P1 } & Compensado & \multirow{9}{*}{0,70} & \multirow{9}{*}{$15 \%$} \\
\hline & & Eucalipto & & \\
\hline & & Pínus & & \\
\hline & \multirow{3}{*}{ P3 } & Compensado & & \\
\hline & & Eucalipto & & \\
\hline & & Pínus & & \\
\hline & \multirow{3}{*}{$\mathrm{P} 1,2$} & Compensado & & \\
\hline & & Eucalipto & & \\
\hline & & Pínus & & \\
\hline & & Ref & 0,70 & $0 \%$ \\
\hline
\end{tabular}

\section{Resultados e discussão}

\section{Análise comparativa das composições granulométricas das partículas de madeira}

Os resultados dos ensaios de determinação da massa unitária C29 (AMERICAN..., 2017) e do teor de umidade D4442 (AMERICAN..., 2016) estão apresentados na Tabela 5. Os resultados de massa unitária seca indicam que, para dosagens realizadas em massa, ocorre uma diminuição no volume de madeira presente nos traços produzidos com as partículas P3 quando comparada às partículas P1 e $\mathrm{P} 1,2$. Isso porque a massa unitária das partículas $\mathrm{P} 3$ é superior às partículas $\mathrm{P} 1$ e P1,2 para todos os tipos de madeira. De modo geral, comparando os três diferentes tipos de madeira, as partículas de compensado $\left(0,148 \mathrm{~g} / \mathrm{cm}^{3}\right.$, em média) apresentaram maior valor de massa unitária seca, seguido pelas partículas de eucalipto $\left(0,144 \mathrm{~g} / \mathrm{cm}^{3}\right.$, em média) e de pínus $\left(0,135 \mathrm{~g} / \mathrm{cm}^{3}\right.$, em média), indicando que compósitos produzidos com compensado tendem a apresentar menor volume de partículas. É importante destacar que a quantidade de partículas de madeira presente nos compósitos é capaz de influenciar seu desempenho mecânico (TURGUT, 2007; MORALES-CONDE; RODRÍGUEZLIÑÁN; PEDREÑO-ROJAS, 2016).

A partir da composição granulométrica das partículas de madeira (Figura 2) é possível observar que a repetição do procedimento de moagem foi eficaz para o aumento da quantidade de partículas finas de eucalipto e pínus. Entretanto, para as partículas de compensado, o mesmo efeito não foi verificado. Isso pode estar relacionado ao fato de que como os resíduos desse tipo de madeira foram coletados em momentos diferentes, pode ter ocorrido uma variabilidade quanto à natureza dessas partículas.

Dentre os tipos de madeira utilizados neste estudo, as composições que contêm a maior quantidade de partículas menores são as de eucalipto, seguida do pínus e, por fim, de compensado. 
Tabela 5 - Caracterização das partículas de madeira

\begin{tabular}{c|c|c|c}
\hline \multicolumn{2}{c|}{ Amostra } & Massa unitária seca $\mathbf{( g / \mathbf { c m } ^ { 3 } )}$ & Teor de umidade (\%) \\
\hline \multirow{3}{*}{ Compensado } & P1 & 0,120 & 8,84 \\
& P3 & 0,209 & 12,67 \\
& P1,2 & 0,115 & 12,95 \\
\hline \multirow{3}{*}{ Eucalipto } & P1 & 0,145 & 8,59 \\
& P3 & 0,163 & 13,53 \\
& P1,2 & 0,125 & 13,86 \\
\hline \multirow{3}{*}{ Pínus } & P1 & 0,138 & 9,14 \\
& P3 & 0,154 & 12,32 \\
& P1,2 & 0,113 & 12,40 \\
\hline
\end{tabular}

Figura 2 - Composição granulométrica das partículas de madeira

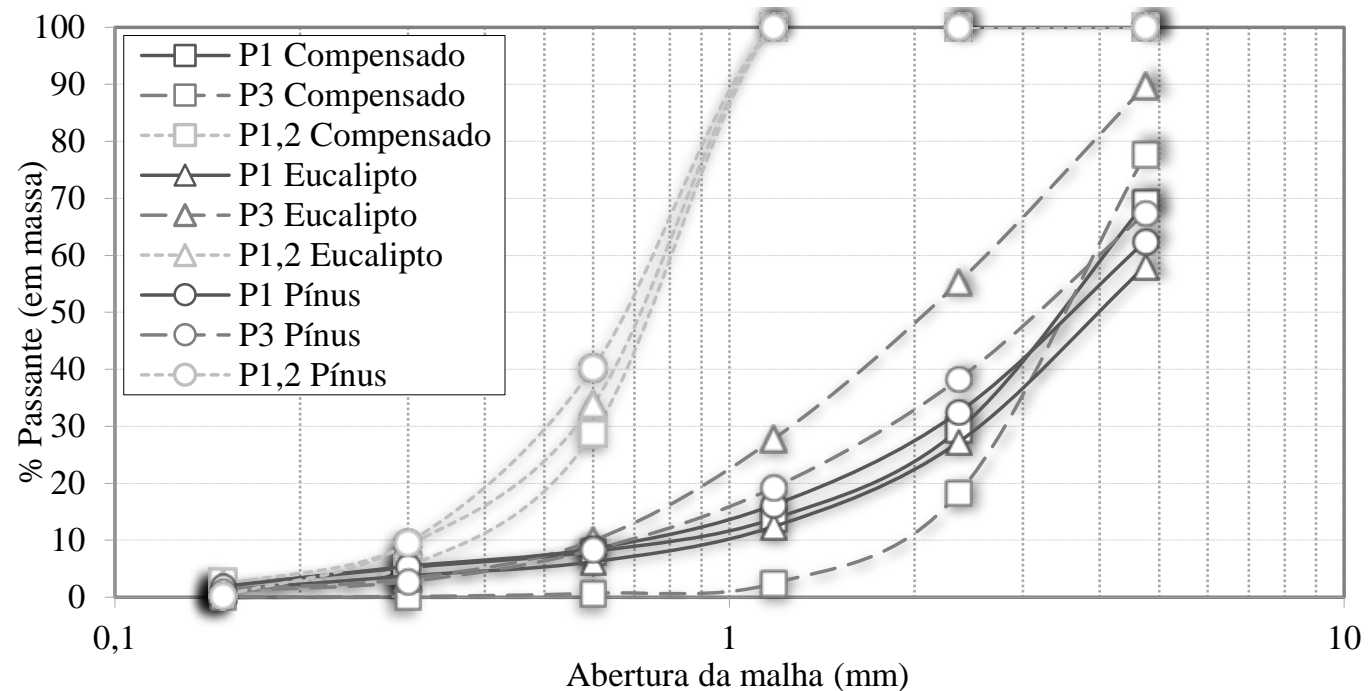

\section{Análise da influência das composições granulométricas das partículas no estado fresco}

Na Figura 3 estão apresentados os resultados obtidos a partir do ensaio de tempo de início e de fim de pega dos compósitos de gesso-madeira. É possível observar que, com relação à composição granulométrica das partículas (P1, P3 e P1,2), tanto o tempo de início de pega como o tempo de fim de pega foram menores para as composições produzidas com as partículas P1,2. Por isso, acredita-se que esse efeito é provocado por um fenômeno físico, isso porque maior fração de partículas com menores dimensões tende a aumentar a área superficial, o que provoca maior absorção de água pela madeira (MORALESCONDE; RODRÍGUEZ-LIÑÁN; PEDREÑOROJAS, 2016). Isso reduz a quantidade de água disponível na mistura, o que contribui para a aceleração da pega do gesso.

Os resultados de densidade de massa fresca (Figura 5) indicam que a composição granulométrica das partículas de madeira exerce pouca influência nesse parâmetro em que os compósitos de gesso apresentaram valores entre $1,49 \mathrm{~g} / \mathrm{cm}^{3}$ e $1,54 \mathrm{~g} / \mathrm{cm}^{3}$. Isso porque a proporção de madeira na composição e a densidade dessas partículas são consideradas pequenas quando comparadas à proporção e à massa específica do aglomerante utilizado (TURGUT, 2007; MORALES-CONDE; RODRÍGUEZ-LIÑÁN; PEDREÑO-ROJAS, 2016). É possível observar, ainda, que a maior densidade de massa fresca foi obtida para as amostras de referência, indicando que a presença de partículas de madeira contribui para a diminuição da densidade dos compósitos (AL RIM et al., 1999; ANDIÇ-ÇAKIR et al., 2014; XIE et al., 2015).

\section{Análise da influência das composições granulométricas das partículas no estado endurecido}

Os resultados de densidade de massa endurecida aos 28 dias dos compósitos de gesso-madeira (Figura 6) evidenciam que os maiores valores foram obtidos para amostras que continham as partículas P3. Isso porque essas partículas, independentemente do tipo de madeira - seja de compensado, de eucalipto ou 
de pínus -, apresentaram os maiores valores de massa unitária seca. Ao realizar a dosagem dos materiais em massa, as partículas com menor massa unitária apresentaram menor volume na mistura, o que contribui para o aumento da densidade (TURGUT, 2007; MORALES-CONDE; RODRÍGUEZ-LIÑÁN; PEDREÑO-ROJAS, 2016).

O mesmo ocorre com as partículas P1,2, cujos valores de massa unitária foram os menores obtidos dentre as composições granulométricas avaliadas e, consequentemente, apresentaram os menores valores de densidade de massa endurecida. Isso porque quanto maior o volume de partículas de madeira na composição menor tende a ser a densidade do compósito (TURGUT, 2007; PEDREÑO-ROJAS et al., 2017).

É importante destacar, ainda, que a análise fatorial indicou equivalência estatística ao considerar como fatores principais o tipo de madeira (compensado, eucalipto e pínus) e a proporção de madeira na composição (7,5\% - 11\% - 15\%). Ou seja, ao considerar como fator o tipo de madeira, foi obtida equivalência estatística os compósitos produzidos com compensado e pínus. Porém, ao considerar como fator a proporção de madeira na composição $(7,5 \%$ - 11\% - 15\%), foi obtida equivalência estatística entre os teores $11 \%$ e $15 \%$.

De modo geral, verificou-se que os compósitos de gesso-madeira produzidos com a composição P1,2, ou com compensado ou pínus, ou com as proporções $11 \%$ ou $15 \%$, tendem a apresentar os menores valores de densidade de massa endurecida.

Por fim, observou-se que as densidades de massa endurecida dos compósitos contendo partículas de madeira foram significativamente inferiores aos resultados obtidos para as amostras de referência.

Figura 3 - Resultado dos tempos de início e fim de pega dos compósitos de gesso-madeira

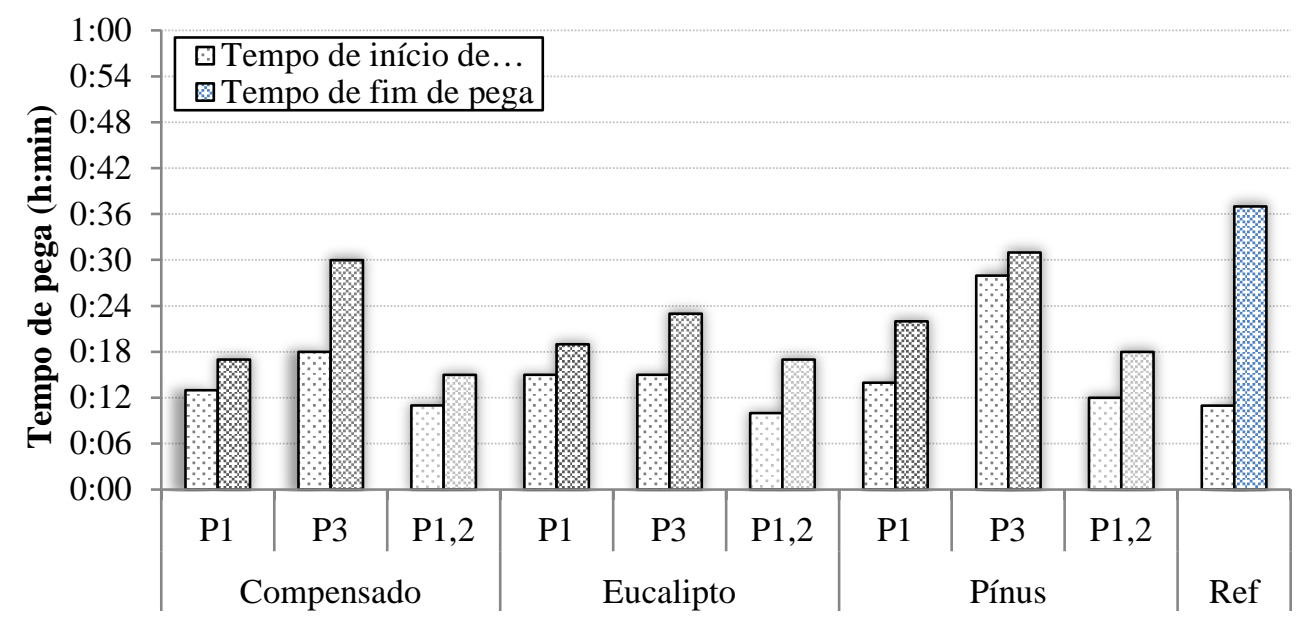

Figura 4 - Resultado da densidade de massa fresca dos compósitos de gesso-madeira

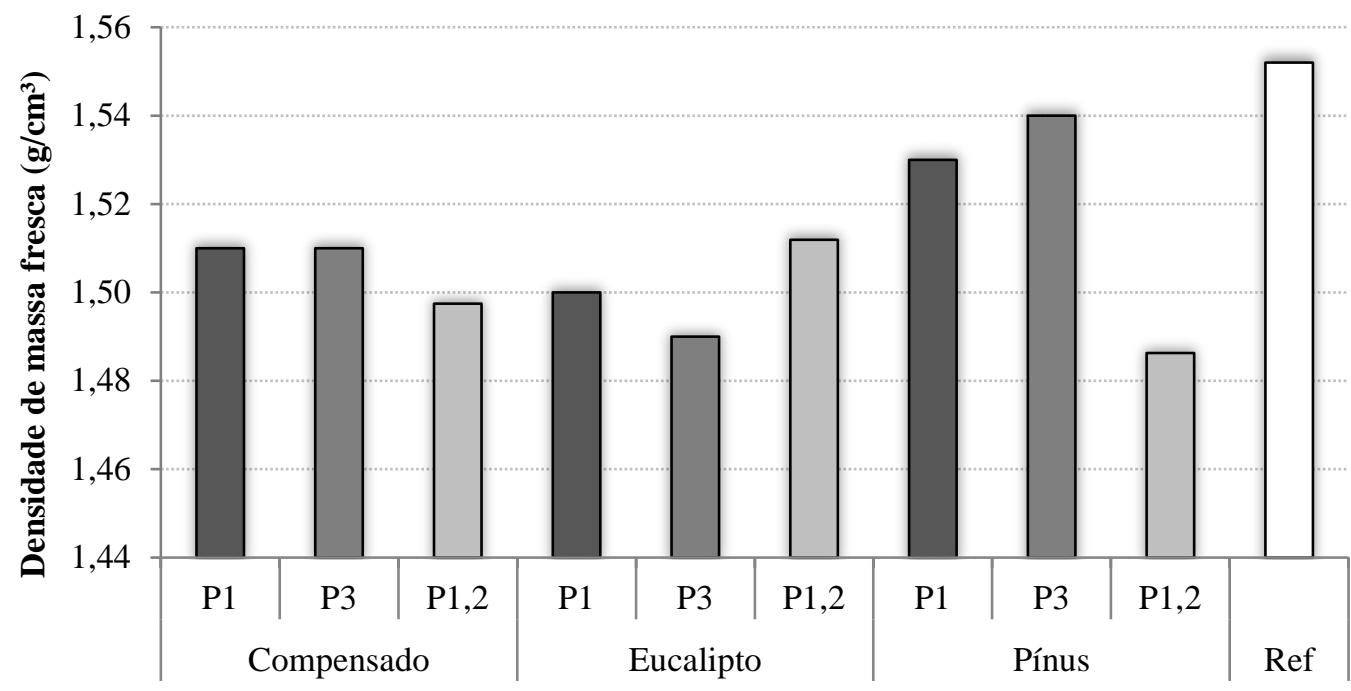


Figura 5 - Resultado da densidade de massa endurecida dos compósitos de gesso-madeira

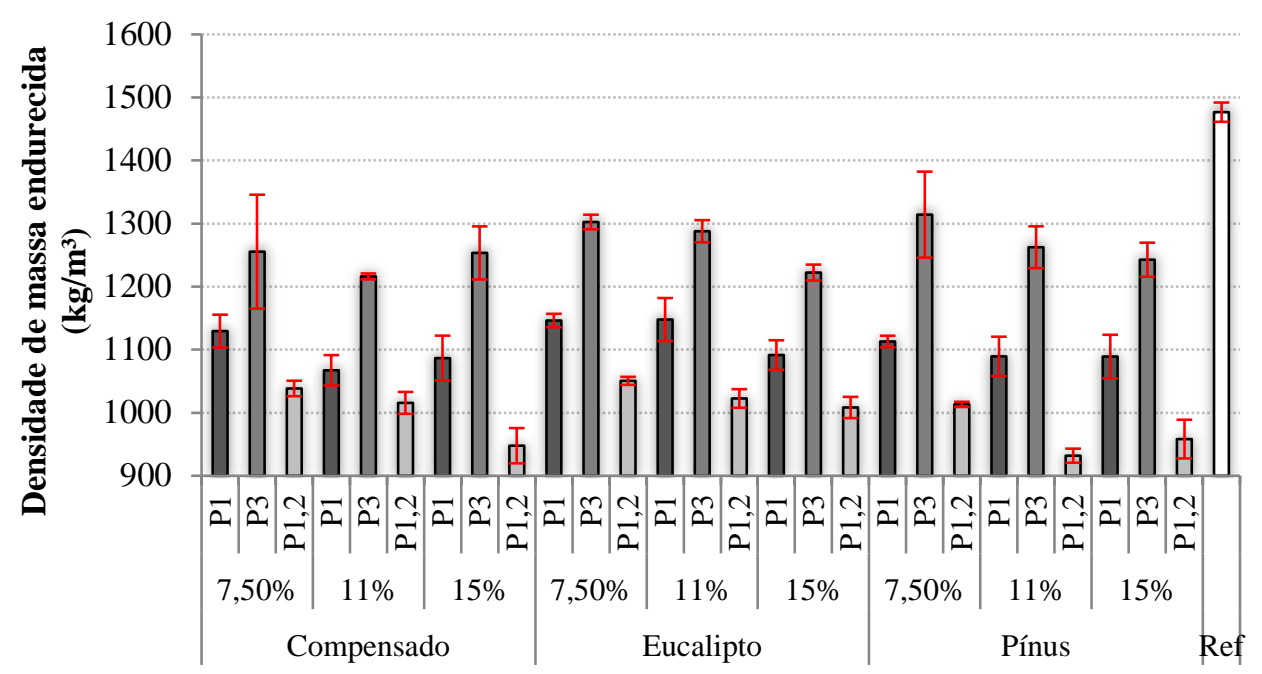

Os resultados de resistência à tração na flexão aos 28 dias dos compósitos de gesso-madeira estão apresentados nas Figuras 6 Observa-se que as menores resistências são obtidas para os compósitos produzidos com as partículas de madeira que contêm a maior quantidade de partículas menores. É importante destacar que compósitos contendo fibras geralmente são macios e dúcteis em torno das fibras - que são geralmente mais fortes — , mas podem ser frágeis em relação à matriz (SMITH, 1994). De acordo com a regra das misturas, cada fase constituinte do compósito contribui para as propriedades efetivas do compósito, sendo essa função dependente apenas da fração volumétrica dos constituintes. Entretanto, essa teoria não considera a presença de vazios nem os efeitos da interface entre a matriz e a carga.

A análise estatística indica, ainda, que ao considerar como fator a proporção de madeira na composição $(7,5 \%$ - 11\% - 15\%), foi obtida equivalência estatística entre os teores $7,5 \%$ e $15 \%$. Para os outros dois fatores avaliados (a composição granulométrica da partícula - P1, P3 e P1,2, e o tipo de madeira - compensado, eucalipto e pínus) foi verificada diferença estatística.

Dessa maneira, os compósitos produzidos com a composição granulométrica $\mathrm{P} 1$, ou com as partículas de eucalipto, ou com as proporções de $7,5 \%$ ou $15 \%$ tendem a apresentar maior resistência à tração na flexão.

Vale destacar, ainda, que os compósitos de gessomadeira produzidos com as partículas de eucalipto na composição granulométrica P1 apresentou resultado de resistência à tração na flexão superior à amostra de referência.

É importante destacar que a discrepância dos resultados dos compósitos produzidos também pode estar relacionada à dispersão não uniforme das partículas de madeira, podendo influenciar o desempenho dos corpos de prova, principalmente quando submetidos ao esforço de tração na flexão (PEDREÑO-ROJAS et al., 2017).

Os resultados de resistência à compressão aos 28 dias dos compósitos de gesso-madeira estão apresentados na Figura 7. É possível observar a influência da proporção de madeira na composição. Isso porque para maiores quantidades de madeira menor tende a ser a resistência à compressão (MORALES-CONDE; RODRÍGUEZ-LIÑÁN; PEDREÑO-ROJAS, 2016). Além disso, devido à diferença dos valores de massa unitária entre as partículas P1, P3 e P1,2, observa-se que os compósitos produzidos com as partículas $\mathrm{P} 1,2$ apresentam as menores resistências à compressão.

A análise estatística indica, ainda, que ao considerar como fator a composição granulométrica da partícula (P1, P3 e P1,2), foi obtida equivalência estatística entre partículas P1 e P1,2, ou seja, igualdade entre esses compósitos. E, também, para o fator de proporção da madeira $(7,5 \%$ - 11\% $15 \%$ ), foi observada equivalência entre as proporções $11 \%$ e $15 \%$. Para o terceiro fator avaliado (tipo de madeira - compensado, eucalipto e pínus), foi obtida diferença estatística.

De modo geral, os compósitos produzidos apresentaram resistência à compressão superior a 2 $\mathrm{MPa}$, indicando que o contato mecânico entre a matriz de gesso e as partículas de madeira foi satisfatório.

De modo geral, verificou-se que os compósitos de gesso-madeira produzidos com as composições granulométricas P1 ou P1,2, ou com eucalipto, ou com a proporção $7,5 \%$ tendem a apresentar os maiores valores de resistência à compressão. 
Figura 6 - Resultado da resistência à tração na flexão dos compósitos de gesso-madeira

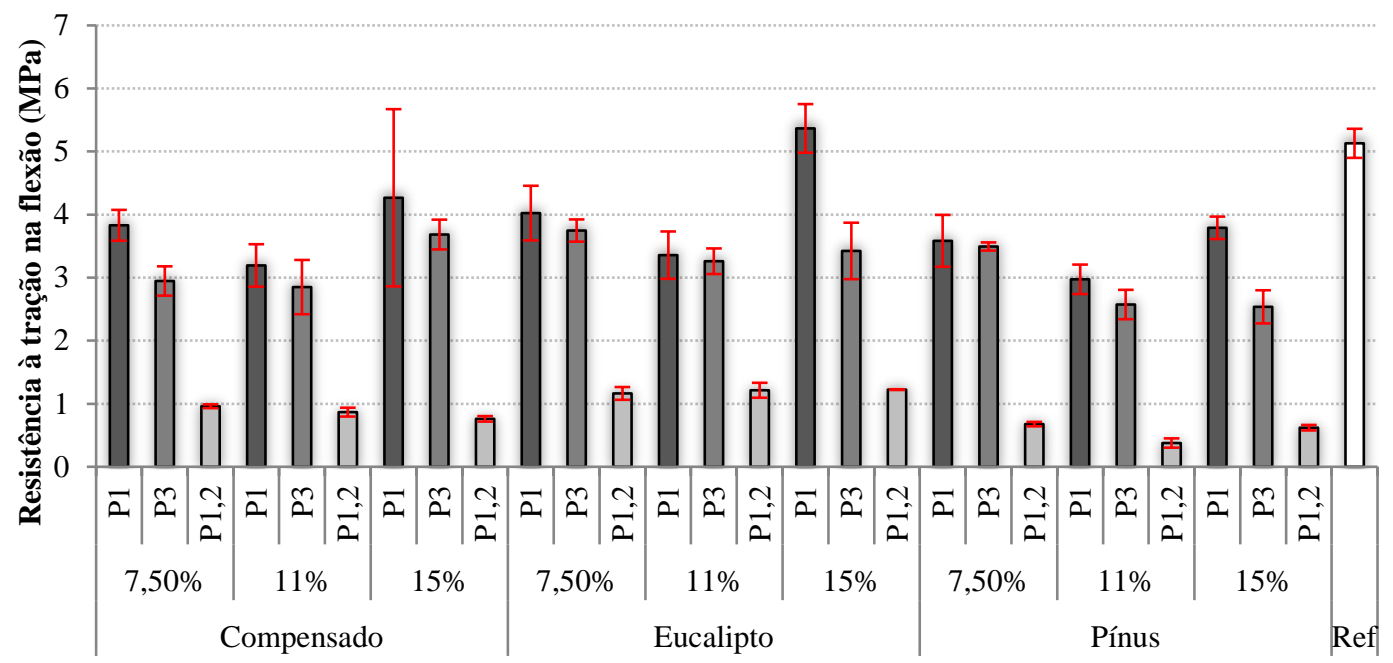

Figura 7 - Resultado da resistência à compressão dos compósitos de gesso-madeira

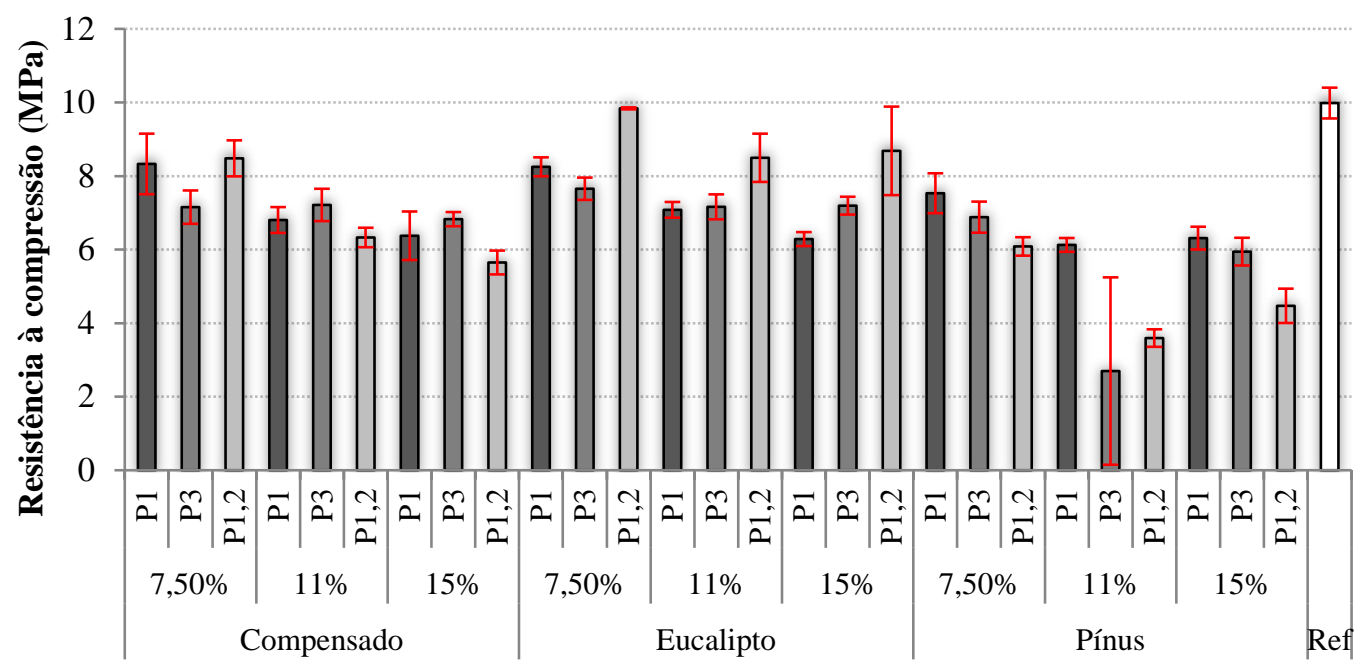

Por fim, observou-se que as resistências à compressão dos compósitos contendo partículas de madeira foram inferiores aos resultados obtidos para as amostras de referência.

\section{Conclusões}

Neste artigo foi verificada a influência da composição granulométrica das partículas de resíduos de madeira nas propriedades de compósitos gesso-madeira. Foi observado, ainda, o efeito de três diferentes tipos de madeira (compensado, eucalipto e pínus) e de três diferentes proporções de madeira na composição $(7,5 \%$ - $11 \%$ - 15\%). Com base nos resultados, as seguintes conclusões foram obtidas: (a) os resultados de massa unitária seca indicam que, para dosagens realizadas em massa, ocorre uma diminuição no volume de madeira presente nos traços produzidos com as partículas moídas três vezes (P3) quando comparada às partículas moídas apenas uma vez (P1) e as partículas passantes na peneira $1,2 \mathrm{~mm}(\mathrm{P} 1,2)$;

(b) os tempos de pega dos compósitos sofreram maior influência quando produzidos com a composição granulométrica P1,2. Isso porque uma maior fração de partículas com menores dimensões tende a aumentar a área superficial, o que provoca maior absorção de água pela madeira, reduzindo a quantidade de água disponível na mistura e contribuindo para a aceleração da pega do gesso;

(c) as maiores densidades de massa fresca e endurecida foram obtidas para amostras de 
referência, o que corrobora com os resultados da literatura que indicam que a presença de partículas de madeira contribui para a diminuição da densidade dos compósitos;

(d) os compósitos de gesso-madeira com as maiores densidades de massa endurecida continham a composição granulométrica P3. Isso porque essas partículas, independentemente do tipo de madeira (seja de compensado, de eucalipto ou de pínus), apresentaram os maiores valores de massa unitária seca e, consequentemente, menor volume de partículas na composição. Em contrapartida, os compósitos de gesso-madeira produzidos com a composição P1,2, ou com compensado ou pínus, ou com as proporções $11 \%$ ou $15 \%$ tendem a apresentar os menores valores de densidade de massa endurecida;

(e) os compósitos de gesso-madeira produzidos com as partículas de eucalipto na composição granulométrica P1 apresentaram resultados de resistência à tração na flexão iguais ou superiores às amostras de referência, diferente dos resultados de resistência à compressão, em que os compósitos contendo partículas de madeira foram inferiores aos resultados obtidos para as amostras de referência; e

(f) os resultados de resistência apresentaram diferentes comportamentos, principalmente de acordo com o tipo de madeira (de compensado, de eucalipto ou de pínus) utilizado para a produção dos compósitos. De modo geral, os compósitos produzidos com a composição granulométrica $\mathrm{P} 1$, ou com as partículas de eucalipto, ou com a proporção de 7,5\% tendem a apresentar maior resistência à tração na flexão e à compressão.

\section{Referências}

AGOUA, E. et al. Thermal Conductivity of Composites Made of Wastes of Wood and Expanded Polystyrene. Construction and Building Materials, v. 41, p. 557-562, 2013.

AKIL, H. M. et al. Kenaf Fiber Reinforced Composites: a review. Materials and Design, v. 32 n.8-9, p. 4107-4121, 2011.

AKINYEMI, A. B.; AFOLAYAN, J. O.; OLUWATOBI, E. O. Some Properties of Composite Corn Cob and Sawdust Particle Boards. Construction and Building Materials, v. 127, p. 436-441, 2016.

AL RIM, K. et al. Influence of the Proportion of Wood on the Thermal and Mechanical Performances of Clay-Cement-Wood Composites. Cement and Concrete Composites, v. 21, p. 269276, 1999.
AMERICAN SOCIETY FOR TESTING AND MATERIALS. C-29: standard test method for bulk density (unit weight) and voids in aggregate.West Conshohocken, 2017.

\section{AMERICAN SOCIETY FOR TESTING AND}

MATERIALS. C-136: standard test method for sieve analysis of fine and coarse aggregates. West Conshohocken, 2014a.

AMERICAN SOCIETY FOR TESTING AND MATERIALS. C-270: standard specification for mortar for unit masonry. West conshohocken, 2014c.

AMERICAN SOCIETY FOR TESTING AND MATERIALS. C-348: standard test method for flexural strength of hydraulic-cement mortars. West Conshohocken, 2014d.

AMERICAN SOCIETY FOR TESTING AND MATERIALS. C-349: standard test method for compressive strength of hydraulic-cement mortars (using portions of prisms broken in flexure). West Conshohocken, 2014e.

AMERICAN SOCIETY FOR TESTING AND MATERIALS. C-472: standard test methods for physical testing of gypsum, gypsum plasters and gypsum concrete. West Conshohocken, 2014b.

\section{AMERICAN SOCIETY FOR TESTING AND}

MATERIALS. D-4442: standard test methods for direct moisture content measurement of wood and wood-based materials. West Conshohocken, 2016.

ANDIÇ-ÇAKIR, O. et al. Physical and Mechanical Properties of Randomly Oriented Coir Fiber-Cementitious Composites. Composites Part B: Engineering, v. 61, p. 49-54, 2014.

ASHOUR, T. et al. Conductivity of Unfired Earth Bricks Reinforcedby Agricultural Wastes With Cement and gyPsum. Energy and Buildings, v. 104, p. 139-146, 2015.

CORINALDESI, V.; MAZZOLI, A.; SIDDIQUE, R. Characterization of Lightweight Mortars Containing Wood Processing By-Products Waste. Construction and Building Materials, v. 123, p. 281-289, 2016.

\section{EUROSTAT. Generation of Waste by Waste Category, Hazardousness and NACE Rev 2 Activity. 2017. Disponível em:} $<$ https://data.europa.eu/euodp/pt/data/dataset/5XK Bv10t6va8j1gKuva25Q>. Acesso em: 18 jan. 2018.

FARAHANI, J. N. et al. Engineering Properties of Lightweight Aggregate Concrete Containing Binary and Ternary Blended Cement. Journal of Cleaner Production, v. 149, p. 976-988, 2017. 
FOREST PRODUCTS LABORATORY. Wood Handbook: wood as an engineering material: wood as an engineering material. Madison: U.S. Department of Agriculture, 2010.

KHORAMI, M.; GANJIAN, E. The Effect of Limestone Powder, Silica Fume and Fibre Content on Flexural Behaviour of Cement Composite Reinforced by Waste Kraft Pulp. Construction and Building Materials, v. 46, p. 142-149, 2013.

LATORRACA, J. V.; IWAKIRI, S.; LELIS, R. C. Efeito Inibidor de Cinco Espécies Florestais Sobre a Cura do Compósito Cimento-Madeira. Floresta e Ambiente, v. 6, n. 1, p. 76-82, 1999.

LATORRACA, J. V. de F. Eucalyptus spp na Produção de Painéis de Cimento-Madeira. Curitiba, 191f. Tese (Doutorado em Engenharia Florestal) - Setor de Ciências Agrárias, Universidade Federal do Paraná, Curitiba, 2000.

LIMA, A. J. M. Utilização de Resíduo de Pinus spp e Materiais Cimentícios Alternativos na Produção de Blocos Para Alvenaria Estrutural. Curitiba, 2009. $281 \mathrm{f}$. Tese (Doutorado em Engenharia Florestal) - Setor de Ciências Agrárias, Universidade Federal do Paraná, Curitiba, 2009.

LIMA, A. J. M.; IWAKIRI, S. Produtos Alternativos na Produção de Blocos Para Alvenaria Estrutural. Floresta e Ambiente, v. 18, n. 3, p. 310-323, 2011.

MAGALLANES-RIVERA, R. X. et al. Modified Gypsum Compounds: an ecological-economical choice to improve traditional plasters.

Construction and Building Materials, v. 37, p. 591-596, 2012.

MEHTA, P. K.; MONTEIRO, P. J. M. Concreto: microestrutura, propriedades e materiais. 2. ed. São Paulo: Ibracon, 2014.

MILLER, S. A.; LEPECH, M. D.; BILLINGTON, S. L. Application of Multi-Criteria Material Selection Techniques to Constituent Refinement in Biobased Composites. Materials and Design, v. 52, p. 1043-1051, 2013.

MORALES-CONDE, M. J.; RODRÍGUEZLIÑÁN, C.; PEDREÑO-ROJAS, M. A. Physical and Mechanical Properties of Wood-Gypsum Composites From Demolition Material in Rehabilitation Works. Construction and Building Materials, v. 114, p. 6-14, 2016.

MOSLEMI A. A.; GARCIA, J. F.; HOFSTRAND, A. D. Effect of Various Treatments and Additives on Wood-Portland Cement Water Systems. Wood and Fiber science, v. 15, n. 2, p. 164-176, 1983.
OSSA, A.; GARCÍA, J. L.; BOTERO, E. Use of Recycled Construction and Demolition Waste (CDW) Aggregates: a sustainable alternative for the pavement construction industry. Journal of Cleaner Production, v. 135, p. 379-386, 2016.

PEDREÑO-ROJAS, M. A. et al. Eco-Efficient Acoustic and Thermal Conditioning Using False Ceiling Plates Made From Plaster and Wood Waste. Journal of Cleaner Production, v. 166, p. 690-705, 2017.

PERVAIZ, M.; SAIN, M. M. Carbon Storage Potential in Natural Fiber Composites. Resources, Conservation and Recycling, v. 39, p. 325-340, 2003.

PIETRINI, M. et al. Comparative Life cYcle Studies on Poly(3- hydroxybutyrate)-Based Composites as Potential Replacement for Conventional Petrochemical Plastics.

Biomacromolecules, v. 8, n. 7, p. 2210-2218, 2007.

ROCHA, T. M. S. Contribuição ao

Desenvolvimento de Placas Para Vedação Vertical Produzidas Com Resíduo de Madeira de Construção, Utilizando Gesso e Cimento Como Aglomerante. Curitiba, 2017. 204 f. Dissertação (Mestrado em Engenharia Civil) Escola de Engenharia, Universidade Federal do Paraná, Curitiba, 2017.

RUMŠYS, D. et al. Comparison of Material Properties of Lightweight Concrete With Recycled Polyethylene and Expanded Clay Aggregates. Procedia Engineering, v. 172, p. 937-944, 2017.

SAVASTANO JUNIOR, H. et al. Plant Fibre Reinforced Cement Components for Roofing. Construction and Building Materials, v. 13, p. 433-438, 1999.

SIMATUPANG, M. H. Der Wasserbedarf bei der Herstellung zementgebunder Holzspanplatten. Holz als Roh-und Werkstoff, v. 27, p. 379-382, 1979.

SMITH, E. H. Mechanical Engineer's Reference Book. Butterworth-Heinemann Ltd., 1994.

TAN, H. et al. Effect of Borax and Sodium Tripolyphosphate on Fluidity of Gypsum Paste Plasticized by Polycarboxylate Superplasticizer. Construction and Building Materials, v. 176, p. 394-402, 2018.

TURGUT, P. Cement Composites with Limestone Dust and Different Grades of Wood Sawdust. Building and Environment, v. 42, p. 3801-3807, 2007. 
VÄNTSI, O.; KÄRKI, T. Environmental Assessment of Recycled Mineral Wool and Polypropylene Utilized in Wood Polymer Composites. Resources, Conservation and Recycling, v. 104, p. 38-48, 2015.

XIE, X. et al. Cellulosic Fibers From Rice Straw and Bamboo Used as Reinforcement of CementBased Composites For Remarkably Improving Mechanical Properties. Composites Part B: Engineering, v. 78, p. 153-161, 2015.
XU, X. et al. Life Cycle Assessment of WoodFibre-Reinforced Polypropylene Composites. Journal of Materials Processing Technology, v. 198, n. 1-3, p. 168-177, 2008.

Thaísa Mariana Santiago Rocha

Programa de Pós-Graduação em Engenharia de Construção Civil | Universidade Federal do Paraná | Centro Politécnico, J ardim das Américas | Curitiba - PR - Brasil | Caixa Postal 19.011 | CEP 81531-980 | Tel.: (41) 3361-3364 | E-mail: thaisamsrocha@yahoo.com.br

Leonardo Fagundes Rosemback Miranda

Programa de Pós-Graduação em Engenharia de Construção Civil | Universidade Federal do Paraná | E-mail: reciclagem.miranda@gmail.com

\section{Carlos Frederico Alice Parchen}

Programa de Pós-Graduação em Engenharia de Construção Civil | Universidade Federal do Paraná | Tel.: (41) 3361-3000 Ramal 3001 | E-mail: parchen.ufpr@gmail.com

\section{Revista Ambiente Construído}

Associação Nacional de Tecnologia do Ambiente Construído

Av. Osvaldo Aranha, 99 - 3o andar, Centro

Porto Alegre - RS - Brasil

$$
\text { CEP } 90035-190
$$

Telefone: +55 (51) 3308-4084

Fax: +55 (51) 3308-4054

www. seer. ufrgs. br/ ambienteconstruido

E-mail: ambienteconstruido@ufrgs.br 